\title{
On the development of a FHIR-compliant backend for processing HTTP requests and API-based management of healthcare documents
}

René Happel, Institute for Biomedical Engineering (IBMT), Faculty of Life Science Engineering (LSE), Technische Hochschule Mittelhessen (THM) - University of Applied Sciences, Gießen, Germany, rene.happel@lse.thm.de Pavel Larionov, Institute for Biomedical Engineering (IBMT), Faculty of Life Science Engineering (LSE), Technische Hochschule Mittelhessen (THM) - University of Applied Sciences, Gießen, Germany, pavel.larionov@lse.thm.de Thomas Schanze, Institute for Biomedical Engineering (IBMT), Faculty of Life Science Engineering (LSE), Technische Hochschule Mittelhessen (THM) - University of Applied Sciences, Gießen, Germany, thomas.schanze@lse.thm.de

\section{Introduction}

In palliative care, it is important to inform relatives or caring persons about the condition of the affected person. Non professional caregivers need information around the topic. This work deals with the task to develop the backend of an app, which could turn in a digital health application (DiGA), where on the one hand a database of articles about palliative care is provided and on the other hand a secure storage place for health data and documents of the affected person is offered.

\section{Methods}

Two RESTful NodeJs server running on Ubuntu were set up so they can be accessed via http-Requests to modify a MongoDB Database. Health data is stored in FHIR-format, to support the exchange between the software systems of the different healthcare stakeholders. Java Web Tokens (JWT) are used for authorization and authentication.

\section{Results}

By using the FHIR-standard, the user-data in the developed app can be easily shared between healthcare stakeholders, giving the secure transfer of it and access to it of cause. The stated need for security was covered with implementation of JWT, which helps to protect all ressource endpoints, from unauthorized requests. The current main feature of the app - the database of articles with bite-size information about palliative care can be shown inside the app and outside of it as webpage, providing the universal and modern way of accessing information.

\section{Conclusion}

The backend implementation for the app for caregivers is a backbone for the future implementation of the cross-institutional bridge for citizen health data. In its current state, the project represents the user-centered approach of handling data, this also applies to backend access, as the user only has control over the flow of this data, and that is how it should be. The articles database can replace traditional media, which are used for information spreading in healthcare - brochures and books. This can address the major lack of healthcare literacy among citizens and empower caregivers and patients, e.g. to use digital health apps.

Sponsored by The Federal Joint Committee of Germany (G-BA). 


\section{Development and Implementation of an Open-Source IoT Platform, Network and Data Warehouse for Privacy-Compliant Applications in Research and Industry}

Sebastian Stadler, Hochschule München University of Applied Sciences, Munich, Germany, sstadler@hm.edu Prof. Dr. Christian Hanshans, Hochschule München University of Applied Sciences, München, Germany, christian.hanshans@hm.edu

Eduardo Romero Borrero, Hochschule München University of Applied Sciences, Munich, Germany, eduardo.romero_borrero@hm.edu

\section{Introduction}

Data privacy and data security are major requirements in the scientific field - especially for medical applications. This is ensured by encrypting data and data streams. However, this alone is not always sufficient if patient-related data is to be persistently stored in the cloud. In addition, the data must be accessible to the doctor and scientist in a user-friendly manner, even from a distance, particularly in the case of geo-distributed studies. Therefore, the IoTree42 platform was developed and tested for these specific requirements.

\section{Methods}

In general, data protection and data security were taken into account during development in the sense of security-bydesign, e.g., by minimizing interfaces, a well-thought-out database design, stable server technology and application-oriented transport encryption. IoTree 42 uses lightweight open-source software cast into a flexible and efficient backend. The data itself is transmitted with low overhead via MQTT protocol, whereby the transmission technology between gateway and devices is freely selectable. It was considered that the platform will not only be used on full-fledged servers, but also on small SBCs like the Raspberry Pi. To minimize the hurdle of integration into an existing infrastructure and to simplify further processing of the collected data, common protocols were used, and an application programming interface (API) was implemented.

\section{Results}

The result is an open source, privacy-compliant, hardware and cloud agnostic IoT data platform with multiple device integration options, designed primarily for use in science and engineering. It consists of communication layer, control software, user management, data storage and visualization tools. Once the platform is in production, new gateways and devices can be added to the network in less than a minute in a self-explanatory way. Complex network architectures with sub-networks and load distribution are just as feasible as a locally decoupled operation. The accruing data can be searched, conveniently visualized in a dashboard, and downloaded for more comprehensive evaluations. If required, patient or subject data can be automatically pseudonymized directly at the gateway level.

\section{Conclusion}

With IoTree42, a wide range of applications can be achieved, from simple data acquisition and automation to complex data analysis for data-intensive scientific studies. In contrast to many commercial alternatives, IoTree42 shows the great advantage of storing valuable data by yourself and adopting the platform to individual needs. Besides the reduced cost potential, users benefit from the freedom to bootstrap the development of remote sensing and remote control in a variety of use cases - from home automation, scientific purposes, up to industrial applications with large scale distributed sensor networks. 


\title{
TeleSDC - Concept for ISO/IEEE 11073 SDC in telemedicine across unreliable networks
}

\author{
Tobias Pabst, ICCAS (Faculty of Medicine, University Leipzig), Leipzig, Germany, Mail ${ }^{1}$ \\ Dominik Stegemann, SurgiTAIX AG, 52134 Herzogenrath, Germany, Lastname@surgitaix.com \\ Christoph Georgi, ICCAS (Faculty of Medicine, University Leipzig), Leipzig, Germany, Mail ${ }^{1}$ \\ Martin Kasparick, Institute of Applied Microelectronics and Computer Engineering (University Rostock), Rostock, Ger- \\ many, Firstname.Lastname@uni-rostock.de \\ Thomas Neumuth, ICCAS (Faculty of Medicine, University Leipzig), Leipzig, Germany, Mail ${ }^{1}$ \\ Max Rockstroh, ICCAS (Faculty of Medicine, University Leipzig), Leipzig, Germany, Mail ${ }^{1}$
}

\section{Introduction}

The standards family IEEE 11073 Service-oriented Device Connectivity (SDC) was introduced to create interoperability between medical devices and to physically separate a device and its control. Based on SDC, several medical devices and Point of Care Units are being developed. In the currently available implementation of SDC it is required that devices share the same local network.

In fields such as emergency or nursing care, the physician and the patient can be physically separated and must rely on telemedicine to support the treatment of the patient. To increase the quality of telemedical support by the physician, SDC can be used to transmit patient and device data to the physician, where it can aid in diagnosis and decision support. In addition, information such as medication or pre-existing illnesses can be obtained from the clinic to improve the treatment of the patient. This, however, requires the SDC devices to communicate over multiple potentially unstable and unreliable, in some cases also public, networks.

\section{Methods}

One way to connect SDC devices located in different networks is to use a VPN, creating a single IP-range. This leads to a set of problems, especially if the network quality is unstable, for example in mobile networks. We analysed these problems and developed requirements for an SDC bridge to enable a safe and consistent connection between multiple SDC networks. Based on these requirements a concept of a system was developed, which is discussed in this work.

This analysis is part of the current ongoing MOMENTUM research project, funded by the German Federal Ministry of Education and Research (BMBF), which aims to interconnect an ambulance and the emergency command centre of a hospital. Through this project we connected with various physicians and medical device manufactures to strengthen our analysis.

\section{Results}

The local and remote networks are connected using an SDC bridge via an arbitrary network route. Since the bandwidth of this route is variable, our concept offers the possibility to prioritize the shared data based on the available bandwidth to provide a meaningful SDC service even in case of a bandwidth shortage.

Our solution aggregates the devices of the local network and mirrors them to the remote network of the telemedicine provider in the sense of a digital twin, to enable full compatibility of the devices according to the SDC standard. SDC devices in the local and remote network can recognize requests and controls from the other network as such and take them into account in their risk management. This also enables, for the first time, a semantic distinction between a local physician and a telemedicine physician at the SDC level.

\section{Conclusion}

We present the requirements for interconnected SDC networks for use in preclinical emergency care. Based on those requirements we concluded a general concept and built and tested a demonstration software to review our concept in a real-world scenario. The MOMENTUM consortium will continue to implement this concept for preclinical emergency care.

\footnotetext{
${ }^{1}$ Firstname.Lastname@medizin.uni-leipzig.de
} 


\section{Service-Oriented Medical Device Connectivity: Particular Interoperability Standards for High Frequency Surgical Equipment and External Control Devices}

Martin Kasparick, Inst. of Appl. Microelectronics, University of Rostock, Germany, martin.kasparick@uni-rostock.de Marcus Köny, steute Technologies GmbH \& Co. KG, Löhne, Germany, m.koeny@steute.com

Björn Andersen, Drägerwerk AG \& Co. KGaA, Lübeck, Germany, bjoern.andersen@draeger.com

Kathrin Riech, Institute of Medical Informatics, University of Lübeck, Lübeck, Germany, k.riech@uni-luebeck.de

Anton Keller, Aesculap AG, Tuttlingen, Germany, anton.keller@aesculap.de

Sven Kämmer, BOWA-electronic GmbH \& Co. KG, Gomaringen, Germany, sven.kaemmer@bowa-medical.com

Andreas Guth, Erbe Elektromedizin GmbH, Tübingen, Germany, andreas.guth@erbe-med.com

Lars Mündermann, Karl Storz SE \& Co. KG, Tuttlingen, Germany, lars.muendermann@karlstorz.com

Axel Stickel, Karl Storz SE \& Co. KG, Tuttlingen, Germany, axel.stickel@karlstorz.com

Stephan Klöckner, Olympus Winter \& Ibe GmbH, Hamburg, Germany, stephan.kloeckner@olympus-oste.eu

Frank Golatowski, Inst. of Appl. Microelectronics, University of Rostock, Germany, frank.golatowski@uni-rostock.de

Dirk Timmermann, Inst. of Appl. Microelectronics, University of Rostock, Germany, dirk.timmermann@uni-rostock.de

\section{Introduction}

Clinical users have a great demand for applications based on manufacturer-independent medical device interoperability. The ISO/IEEE 11073 Service-oriented Device Connectivity (SDC) series of standards is a key enabling technology. The SDC core standards have been published and adopted. The first SDC-based products are used in patient care. However, there is a strong need for extending the SDC family with Device Specializations (DevSpecs) - particular interoperability standards that describe the network behavior of complex medical devices to ensure safe interoperability.

\section{Methods}

Developing Device Specializations is a challenging task: The model has to cover all current and foreseeable medical devices of one type, including their parameters, measurements, functionalities, etc. The standard has to be as strict as necessary to reduce the implementation complexity of service providers and consumers, but it also has to be as flexible as possible to allow manufacturer-specific innovations. We therefore initiated the research project Modular Specializations for Point-of-Care Medical Devices (PoCSpec) funded by the German Federal Ministry for Economic Affairs and Energy(BMWi). Based on the broad expertise of the device manufacturers' domain experts and interoperability experts, we analyzed the interconnection-related aspects of high frequency (HF) medical equipment and its foot switch used for activation of the HF generator. Foot switches have been generalized to external control devices. We transferred this knowledge to comprehensive device type models by using consensus principles, which are common in standards development.

\section{Results}

We propose a model for HF surgical equipment that covers all relevant elements for network representation: The two basic user interaction approaches, active accessory-oriented and active output terminal-oriented, are considered as well as aspects like bipolar and monopolar application, neutral electrode, argon gas supply, programs, or user interface settings. Our work on external control devices takes different kinds of user interaction items into account: foot switches, finger switches, buttons, joysticks, rocker switches, etc. Such items are used by different medical devices. Thus, we introduce the concept of Module Specifications (ModSpecs) that can be utilized by other standards or implementations. As HF surgical devices typically have configurable foot switches, the respective DevSpec utilizes the corresponding aspects of the ModSpec standard.

\section{Conclusion}

This work adds new standard proposals to the IEEE 11073 SDC family of standards: IEEE P11073-10721 (DevSpec for HF surgical equipment) and parts of IEEE P11073-10720 (including ModSpec for external control items). Additionally, we have created several hundred nomenclature terms to be standardized in IEEE P11073-10101c, which is an extension of the well-established IEEE 11073-10101 nomenclature. These standardization efforts address one of the last open interoperability challenges for complex medical devices. Thus, this work directly supports the manufacturers and is one key enabler to transfer the technology for manufacturer-independent interoperability from research to the market in order to improve patient safety, clinical outcome, and clinical workflows. 


\section{Generation of surgical reports using keyword-augmented next se- quence prediction}

Richard Bieck, Innovation Center Computer Assisted Surgery, University Leipzig, Leipzig, Germany, Richard.bieck@ic$\underline{\text { cas.de }}$

Valentina Wildfeuer, ENT Department, Leipzig Medical Center, Leipzig, Germany

Viktor Kunz, ENT Department, Leipzig Medical Center, Leipzig, Germany

Martin Sorge ENT Department, Leipzig Medical Center, Leipzig, Germany

Markus Pirlich, ENT Department, Leipzig Medical Center, Leipzig, Germany

Thomas Neumuth, Innovation Center Computer Assisted Surgery, University Leipzig, Leipzig, Germany

\section{Introduction}

The documentation of a surgical procedure remains a time-consuming task that surgeons have to incorporate into their daily routine. However, since a surgical report should be produced immediately after the operation with all impressions of the procedure in mind, a means of automation assistance should be provided. We, therefore, propose a method that generates surgical reports based on keywords stated during the procedure.

\section{Methods}

Our report generation is based on a sequence-to-sequence model that is trained on sentence pairs of two consecutive sentences in a surgical report. The known sentence is augmented with a keyword based on the following surgical action to be documented and is then passed into a language model to generate the next sentence. In this way, the complexity of predicting a vast number of possible surgical report phrasings is reduced to a next sentence prediction task. For the language model an encoder-decoder-structure was used with bidirectional 2-layer LSTM units for both components and an attention layer between input and output sentences. The training data consisted of 50 ENT reports with 1500 sentences. The model training was performed in a $\mathrm{k}$-fold cross-validation study with $\mathrm{k}=10$ and cross-entropy loss as the objective function. The generated reports were investigated using NIST, ROUGE and METEOR metrics. Additionally, medical experts identified the report content regarding plausibility.

\section{Results}

The trained models reached an accuracy of 0.82 for next sentence predictions. The generated reports show consistent sentence structures and keyword correspondence for about $70 \%$ of provided keyword sequences. The NIST, ROUGE and METEOR metrics reached $0.65,0.71$ and 0.64 , respectively. The model underperformes for unknown keyword sequences and shows signs of overfitting when keyword sequences deviate from the baseline of the training set.

\section{Conclusion}

Our approach for the keyword-augmented generation of surgical reports shows the potential of reducing the text generation complextiy by providing a sequence of anchor words. However, the automated generation of surgical reports remains a difficult task due to individual report phrasings and the high variance in keyword sequences. 


\section{The Digital Twin: Modular Model-Based Approach to Personalized Medi- cine}

Jan Gaebel, University Leipzig, Faculty of Medicine, ICCAS, Leipzig, Germany, jan.gaebel@medizin.uni-leipzig.de Johannes Keller, University Leipzig, Faculty of Medicine, ICCAS, Leipzig, Germany Daniel Schneider, University Leipzig, Faculty of Medicine, ICCAS, Leipzig, Germany Adrian Lindenmeyer, University Leipzig, Faculty of Medicine, ICCAS, Leipzig, Germany Thomas Neumuth, University Leipzig, Faculty of Medicine, ICCAS, Leipzig, Germany Stefan Franke, University Leipzig, Faculty of Medicine, ICCAS, Leipzig, Germany

\section{Introduction}

Clinical decision making is a complex task with heterogeneous requirements depending on the medical subject. It involves experts from different domains and uses various tools. Large amounts of fragmented patient data, a multitude of clinical guidelines and a vast number of clinical studies need to be considered. Furthermore, clinical information is integrated into a mental model by each physician in the course of clinical decision making. Yet, usually they are left on their own devices with no technical assistance. Altogether, this leads to a series of problems such as incomplete information due to only partial access to data and limited temporal as well as cognitive facilities, biased models.

\section{Methods}

In this paper, we address the above-mentioned problems and present a universal approach to building a predictive diseaseand patient-specific therapeutic decision support system. The digital twin, as a computable representation of a patient with all relevant characteristics, is built as an interlinked RDF graph capable of depicting causal relationships between different entities and describing processing pipelines. It contains aggregated data from electronic health records and knowledge derived from medical textbooks, clinical studies, and therapeutic guidelines. Surrounding the central digital twin, there is a framework of service-oriented modules. This clinical data processing as a service provides data preparation, calculations of medical scores or even evidence-based simulations. Specialised applications systems utilize the twin to provide functionality to physicians and other healthcare professionals.

\section{Results}

The central digital twin is crosslinking modules based on posed clinical questions. New modules can be added, when a new clinical use case arise. Linking different modules together is activating necessary data pre-processing or collecting data from heterogeneous sources. Finally, at the end of a proposed pipeline, a clinical question gets answered and the results can be made available to the clinical user. The reasoning and traceability of the results can also be made available by chaining the steps of the pipeline and presenting them to increase the trust in the digital twin.

\section{Conclusion}

The digital twin is capable of providing patient-specific decision support for patient-specific personalized medicine. Its modular system of independent, interlinked modules allows for distinct processing pipelines. Relevant data entities can be made available for calculations (e.g., treatment suitability) and the depiction of the patient or his or her characteristics becomes even more specific. Not only can decisions and procedures be sounder and physicians' workflow be more efficient. Actions will be tailored more to the individual patients, and the safety of the treatments will increase, as well. 